\title{
Securing while Sampling in Wireless Body Area Networks with Application to Electrocardiography
}

\author{
Ruslan Dautov, Member, IEEE and Gill R Tsouri, Member, IEEE
}

\begin{abstract}
Stringent resource constraints and broadcast transmission in wireless body area network raise serious security concerns when employed in biomedical applications. Protecting data transmission where any minor alteration is potentially harmful is of significant importance in healthcare. Traditional security methods based on public or private key infrastructure require considerable memory and computational resources and present an implementation obstacle in compact sensornodes. This paper proposes a lightweight encryption framework augmenting Compressed Sensing with Wireless Physical Layer Security. Augmenting compressed sensing to secure information is based on the use of the measurement matrix as an encryption key and allows for incorporating security in addition to compression at the time of sampling an analog signal. The proposed approach eliminates the need for a separate encryption algorithm as well as the pre-deployment of a key thereby conserving sensor-node's limited resources. The proposed framework is evaluated using analysis, simulation and experimentation applied to a wireless electrocardiogram setup consisting of a sensor node, an access point, and an eavesdropper performing a proximity attack. Results show that legitimate communication is reliable and secure given that the eavesdropper is located at a reasonable distance from the sensor node and the access point.
\end{abstract}

Index Terms-Compressed sensing, electrocardiography, wireless body area networks, wireless physical layer security

\section{INTRODUCTION}

$\mathbf{W}$ IRELESS Body Area Network (WBAN) is a group of sensors with wireless communication capabilities that are placed on a human body for physiological monitoring and diagnosis of health conditions. Sensor nodes in WBANs are required to be compact, unobtrusive and energy efficient in order to achieve a practical and wearable system that lasts for sufficient time. These requirements impose stringent limitations on resources such as memory size, computation and transmission capabilities. WBANs are expected to proliferate in the near future, following a recent allocation of a dedicated ISM frequency band of 2360-2400 MHz.

Wireless transmission makes WBANs prone to interception and eavesdropping. This security problem significantly affects the deployment of WBANs in biomedical applications where privacy is required by the Security and Privacy rules of the Health Insurance Portability and Accountability Act [1]. Health care providers must ensure confidentiality, integrity and availability of an individual's health records.

Securing information in a resource constrained environment is not trivial. Traditionally, the problem is approached by using

R. Dautov is with the College of Computing and Information Sciences $P h D$ program, Rochester Institute of Technology, Rochester, NY, 14623 USA e-mail: rid6541@ rit.edu

G. R. Tsouri is with the Department of Electrical and Microelectronics Engineering, Rochester Institute of Technology, Rochester, NY, 14623 USA e-mail: grteee@ rit.edu

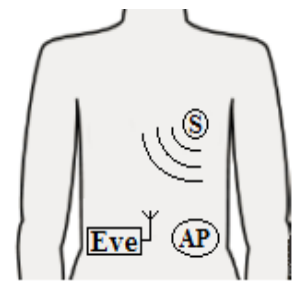

Fig. 1. Simple Wireless Body Area Network in Electrocardiography (S sensor node, AP - access point, Eve - eavesdropper).

Advanced Encryption Standard (AES) [2] along with DiffieHellman key establishing algorithm [3]. Even though this approach provides excellent security and is more energy efficient than widely accepted public key infrastructure algorithms, it was designed for systems with abundant resources. In other words, the computational overhead introduced by AES has a noticeable impact reflected in shortened network lifetime and a need for substantial memory and computational power.

In this paper, we propose a lightweight encryption framework for augmenting Compressed Sensing (CS) [4]-[6] with Wireless Physical Layer Security (WPLS). The framework allows incorporating security in addition to compression while sampling an analog signal using CS. Since there is no need for a separate encryption algorithm, the proposed method imposes marginal overhead and offers all benefits of CS.

The ability of CS to ensure security received some attention in the past [7]-[9]. It is based on using the measurement matrix as a symmetric key for encryption and decryption. However, the problem of key distribution in these works is not addressed and remains unsettled. To avoid the need for resource hungry key distribution (Diffie-Hellman for example) we employ WPLS along with Linear Feedback Shift Register (LFSR). WPLS is an active area of research [10]-[14]. In principle, it aims to extract a common secret between legitimate communicating parties through public discussion over the wireless channel by exploiting intrinsic randomness of the channel. Among the variety of WPLS algorithms we focus on low complexity algorithms where secret bits are retrieved from Received Signal Strength Indicator (RSSI) values. RSSIs are available to use in all commercial transceivers from packets going back and forth between communicating parties. See also works [10], [11] for more examples on WPLS related algorithms, [12] for the limitation of key extraction under a proximity attack and [13] for the intrinsic ability of wireless fading channels to secure information.

A simple WBAN shown in Fig. 1 is considered. It consists of a single sensor node, an access point that resides either on or off the body, and an eavesdropper listening to their 
conversation. Secret bits are extracted from RSSI values of incoming packets. These bits are used as seeds to feed a LFSR to generate an m-sequence. The output m-sequences are later reorganized to form a CS sensing matrix by transmitter and receiver without coordination. A preliminary investigation of the proposed method was provided in [14]. The work considered transmission of synthetic binary data over a Rician fading channel in the presence of eavesdropper performing a proximity attack. By means of simulation and estimated Mutual Information (MI), it was revealed that generated sensing matrices ensure high quality reconstruction and therefore suitable for CS. It was also shown that the adversary is unable to recover the original signal while being physically close to one of the legitimate participants.

In this contribution, we propose a secure CS framework applied to the growing field of wireless biomedical applications. Specifically, we evaluate the performance of the proposed framework on real world ElectroCardioGram (ECG) recordings provided by the MIT Arrhythmia Database [15] using simulations and an experimental setup. To provide more extensive analysis of reconstruction quality we assess widely used similarity metrics of Root Mean Square Error (RMSE), Relative Error (RE), and Similarity Coefficient (SC). In addition, we analyze relative time complexity and security strength against basic attacks. Note that the method of estimation of mutual information provided in [14] is different than the method used in this contribution due to limited source data. To compensate for possible data undersampling, we employ a James-Stein estimator of MI [16]. As in [14] we assume a passive eavesdropper capable of carrying out a proximity attack to reduce spatial selectivity of the Rician fading channel.

\section{COMpressed SENSING}

CS emerged from the pioneering works of Candes, Tao, and Donoho [4]-[6] as a new signal processing paradigm. It allows for perfect or optimal reconstruction of the analog signal from fewer samples than it is suggested by the Nyquist theorem. This means that compression can be achieved while sensing the analog signal. Reduced number of acquired samples is beneficial for WBAN. It mitigates the requirements imposed on sensor storage and processing capabilities.

The signal is said to exhibit sparsity if its "information rate" is much lower than its bandwidth. Sparsity simply reflects the inherent ability of the signal to be compressed. Most physiological signals captured by WBANs have been proved to have a domain with efficient representation. Thus, wavelets are shown to be particularly useful in ECG applications [17]. Without loss of generality we employ CS in discrete-time domain where the field has already reached its maturity.

Considering canonical CS, the signal of interest $x$ can be represented in some domain $\Psi$ comprised of orthogonal set of $N \times 1$ vectors $\left\{\psi_{i}\right\}_{i=1}^{N}: x=\sum_{i=1}^{N} s_{i} \psi_{i}=\Psi S$. Here $S$ is a $N \times 1$ vector of coefficients. Thus, the signal $x$ is called $k$ sparse if its representation above has exactly $k(k<<N)$ nonzero coefficients $s_{i}$. Exact sparsity allows for perfect signal recovery but is rarely achievable for natural signals. More realistic scenario is when the signal $x$ is compressible with only $k$ large and $N-k$ small coefficients. CS can cope with this situation and provide optimal signal reconstruction with small and bounded error.

The sampling procedure of real, finite-length, sparse $N \times 1$ signal $x$ in canonical CS is given by:

$$
y=\Phi x
$$

where $\Phi$ is an $M \times N(M<N)$ matrix called the measurement or sensing matrix and $y$ is a sensed signal of size $M \times 1$. CS is capable of finding a solution for problem (1) when the sensing matrix $\Phi$ satisfies the Restricted Isometry Property (RIP):

$$
\left(1-\delta_{k}\right)\|x\|_{2}^{2} \leq\|\Phi x\|_{2}^{2} \leq\left(1+\delta_{k}\right)\|x\|_{2}^{2}
$$

for all $k$-sparse vectors $x$ and some constant $0<\delta_{k}<1$.

Knowledge of the compressed measurements $y$ and the sensing modality $\Phi$ gives the original signal $x$ in sparsifying domain $\Psi$ after solving the optimization problem:

$$
\min _{\hat{S}}=\|\hat{S}\|_{1} \text { subject to } y=\Phi \Psi \hat{S}
$$

that finds vector $\hat{S}$ with the lowest $L_{1}$ norm. Compared to sampling (1), the reconstruction problem (3) is computationally intensive. However, this fits perfectly into a typical WBAN setup based on star topology where simple sensors communicate with a more powerful access point.

\section{PROPOSED ENCRYPTION FRAMEWORK}

A block-diagram of the proposed encryption framework is presented in Fig. 2. It defines four principal components namely Secret Bits Distillation, LFSR, Sensing Matrix Generation, and CS Encryption. Following is a detailed description of each block and its role in the encryption/decryption process.

\section{A. Secret Bits Distillation}

The block objective is to extract a shared secret between a sensor node and an access point. For this purpose, we exploit the random nature and uniqueness of the wireless channel between two legitimate communicating parties. There are many WPLS algorithms that can be employed here. Thus, in simulation we use a low complexity algorithm that follows [12] and performs the reciprocal single bit quantization of RSSI values. In the experiment, due to correlation of RSSIs in time, the algorithm from [18] is employed. Detailed description of both is given in the corresponding paragraphs of section IV. The output is a binary sequence of length $m$.

\section{B. LFSR}

The LFSR is a shift register whose input is a linear function of register's previous state. The $m$-stage LFSR takes a binary sequence of length $m$, called a seed, shifts it into adjacent positions producing a single output bit and fills the empty position on the other end according to linear feedback. The most commonly used feedback function is exclusive-or (XOR) of some bits in the register. Due to finite number of possible states the LFSR output sequence is also finite and after some point replicates itself. The carefully chosen linear feedback 


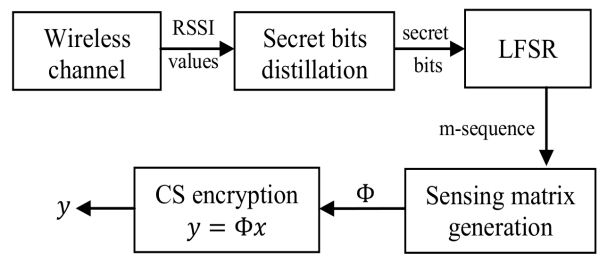

Fig. 2. Block diagram of the proposed encryption framework

based on primitive polynomial allows generating a so-called maximal length sequence or $\mathrm{m}$-sequence. $\mathrm{m}$-sequence has the longest possible repeating cycle of $2^{m}-1$ and features statistical properties that resemble a truly random binary sequence. This fact is crucial for our purposes since we use LFSR for spreading the distilled secret across a longer bit sequence. A set of $\mathrm{m}$-sequences is used to generate a sensing matrix $\Phi$.

\section{Sensing Matrix Generation}

To balance the number of zeros and ones the input $\mathrm{m}$ sequence is augmented with additional zero. Then the elements of a new sequence $\{0,1\}$ are mapped to $\{-1,1\}$ and reorganized to form a matrix. The process is repeated $L$ times for different $\mathrm{m}$-sequences and results in a set of matrices $\Phi_{1}, \Phi_{2}, \ldots, \Phi_{L}$. Arithmetic sum of $\Phi_{i}$ gives the sensing matrix as $\Phi=$ $\sum_{i=1}^{L} \Phi_{i}$. It follows that each entry of the sensing matrix converges to random variable with Gaussian distribution as $L$ increases by the central limit theorem. Note that larger values of parameter $L$ require more bits to be extracted from the channel and thus $L$ defines the amount of randomness in the system. In our preliminary work [14] we also defined a generation of a sensing matrix $\Phi=\prod_{i=1}^{L} \Phi_{i}$ with Bernoulli distribution. However, entry wise matrix multiplication preserves the linearity property of LFSR resulting in reduction of key space to as much as a length of a seed. Therefore, Bernoulli matrices were excluded from further consideration.

\section{CS Encryption/Decryption}

This block is the only part of the framework that differs for transmitter and receiver. Transmitter follows equation (1) and performs secure compressed sampling of the original signal $x$ with matrix $\Phi$. The sensed version $y$ is now safe for transmitting to a receiver over an insecure broadcast channel. On the other end, the receiver solves a convex optimization problem (3) in order to recover the desired signal $x$.

\section{Evaluation Framework AND Metrics}

The evaluation framework consists of three parts which reflect different properties of the proposed encryption method. The first part describes the methodology of analyzing the quality of reconstruction. The second and third parts address time complexity and security aspects respectively.

\section{A. Quality of reconstruction}

1) System Description: To evaluate the quality of reconstruction of the proposed framework we perform simulation

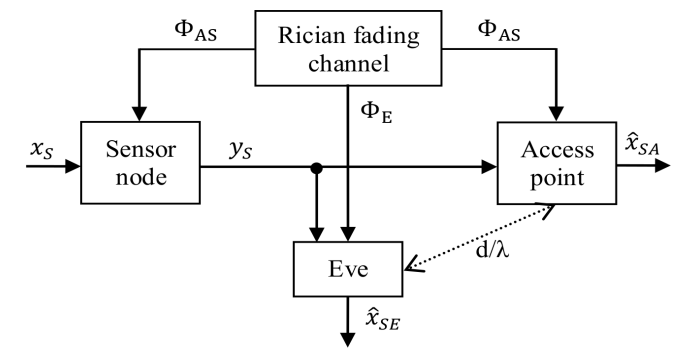

Fig. 3. Block diagram of the performance evaluation framework

along with experimental study. Both employ a wireless ECG scenario depicted in Fig. 3 where a sensor node communicates with an access point via wireless channel. The analysis also includes an adversary party Eve who intercepts legitimate packets and tries to decipher the information. Other than eavesdropping, we assume Eve is a passive adversary.

The communication is initialized by exchanging ordinary packets resulting in RSSI values between the sensor node and the access point. After sufficient number of packets the sensor and the AP generate a CS measurement matrix $\Phi_{A S}$ according to the proposed method. The sensor node applies secure CS (1) to an ECG signal denoted as $x_{S}$ and transmits the result $y_{S}$ to the access point. Having the matrix $\Phi_{A S}$ and measurements $y_{S}$, the access point solves the optimization problem (3) and recovers the original ECG as $\hat{x}_{S A}$. Using the proposed method, Eve aims to extract the sensing matrix $\Phi_{E}$ similar to $\Phi_{A S}$ by performing a proximity attack on the access point. For convenience and generality we normalize distance $d$ between the AP and Eve by communication wavelength $\lambda$ and use the normalized distance $d / \lambda$. The matrix $\Phi_{E}$ along with the intercepted signal $y_{S}$ allows Eve to run the same optimization problem and reconstruct the original signal as $\hat{x}_{S E}$.

As was done in other ECG reconstruction work [19] we evaluate the quality of reconstruction using SC, RE, RMSE. In addition, we estimate MI which reflects security strength of the system and is therefore discussed in IV-C.

2) Simulation Setup: In simulation, the wireless channel is modeled as a distance-based Rician fading channel implemented based on the model reported in [20] following the setup in [18]. The Rician fading occurs when one of the multipath components is stronger than the others which was shown to be an applicable assumption in many practical applications including WBANs. It uses a single parameter called $K$-factor to determine the strength of the dominant multipath component relative to the rest of the multipath components. We assume no noise in the model and thus $\Phi_{A S}$ is always equal for legitimate participants. It is also assumed that simulated RSSI values are not correlated in time.

The WPLS algorithm in simulation setup is derived from [12] and works as follows. Consecutive RSSI estimates are grouped into a sequence of length $m$. After the $m$-th estimate is obtained, the average of the sequence is subtracted from each element and the signs of the elements are then taken to form a binary output seeding the LFSR. The process is repeated until the desired number of sequences $L$ is generated.

We conduct a set of simulation experiments applied to 
TABLE I

RECONSTRUCTION OF AP AND EVE FOR DIFFERENT COMBINATIONS OF $d / \lambda$ AND $K$

\begin{tabular}{|c|c|c|c|c|c|c|c|c|c|}
\hline \multirow{2}{*}{$d / \lambda$} & \multirow{2}{*}{ K } & \multicolumn{2}{|c|}{ MI } & \multicolumn{2}{|c|}{ SC } & \multicolumn{2}{|r|}{$\mathrm{RE}$} & \multicolumn{2}{|c|}{ RMSE } \\
\hline & & mean & variance & mean & variance & mean & variance & mean & variance \\
\hline \multirow{4}{*}{0.01} & 0 & $2.86(0.23)$ & $0.22(0.02)$ & $0.996(0.49)$ & $9.94 \mathrm{E}-6(0.02)$ & $0.090(1.03)$ & 9.9E-4(0.04) & $5.24(66.06)$ & $2.58(984.9)$ \\
\hline & 1 & $2.86(0.31)$ & $0.24(0.16)$ & $0.996(0.53)$ & $1.15 \mathrm{E}-5(0.03)$ & $0.089(0.97)$ & $1.08 \mathrm{E}-3(0.066)$ & $5.20(62.68)$ & $2.33(1217.3)$ \\
\hline & 3 & $2.86(0.39)$ & $0.22(0.29)$ & $0.996(0.56)$ & $1.04 \mathrm{E}-5(0.028)$ & $0.089(0.93)$ & $1.01 \mathrm{E}-3(0.064)$ & $5.23(61.53)$ & $2.63(1176.9)$ \\
\hline & 10 & $2.86(0.32)$ & $0.23(0.26)$ & $0.996(0.51)$ & $1.11 \mathrm{E}-5(0.030)$ & $0.089(0.99)$ & $1.06 \mathrm{E}-3(0.065)$ & $5.24(65.24)$ & $2.57(1266.2)$ \\
\hline \multirow{4}{*}{0.05} & 0 & $2.86(0.035)$ & $0.22(8.6 \mathrm{E}-4)$ & $0.996(0.09)$ & $9.94 \mathrm{E}-06(0.003)$ & $0.090(1.48)$ & $9.9 \mathrm{E}-4(0.004)$ & $5.24(96.65)$ & $2.58(1884.1)$ \\
\hline & 1 & $2.86(0.038)$ & $0.24(0.0011)$ & $0.996(0.10)$ & $1.15 \mathrm{E}-5(0.005)$ & $0.089(1.48)$ & $1.08 \mathrm{E}-3(0.006)$ & $5.20(96.06)$ & $2.33(1784.1)$ \\
\hline & 3 & $2.86(0.036)$ & $0.22(0.0016)$ & $0.996(0.08)$ & $1.04 \mathrm{E}-5(0.006)$ & $0.089(1.50)$ & $1.01 \mathrm{E}-3(0.008)$ & $5.23(97.85)$ & $2.63(1923.1)$ \\
\hline & 10 & $2.86(0.036)$ & $0.23(0.0012)$ & $0.996(0.09)$ & $1.11 \mathrm{E}-5(0.004)$ & $0.089(1.49)$ & $1.06 \mathrm{E}-3(0.005)$ & $5.24(96.85)$ & $2.57(1853.6)$ \\
\hline \multirow{4}{*}{0.1} & 0 & $2.86(0.025)$ & $0.22(7.2 \mathrm{E}-4)$ & $0.996(0.011)$ & $9.94 \mathrm{E}-6(7.07 \mathrm{E}-4)$ & $0.090(1.57)$ & $9.9 \mathrm{E}-4(1.2 \mathrm{E}-3)$ & $5.24(101.7)$ & $2.58(1970.5)$ \\
\hline & 1 & $2.86(0.025)$ & $0.24(7.7 \mathrm{E}-4)$ & $0.996(0.010)$ & $1.15 \mathrm{E}-5(5.57 \mathrm{E}-4)$ & $0.089(1.57)$ & $1.08 \mathrm{E}-3(0.66 \mathrm{E}-3)$ & $5.20(101.6)$ & $2.33(1923.7)$ \\
\hline & 3 & $2.86(0.025)$ & $0.22(7.4 \mathrm{E}-4)$ & $0.996(0.013)$ & $1.04 \mathrm{E}-5(4.98 \mathrm{E}-4)$ & $0.089(1.57)$ & $1.01 \mathrm{E}-3(0.67 \mathrm{E}-3)$ & $5.23(101.7$ & $2.63(2050.3)$ \\
\hline & 10 & $2.86(0.025)$ & $0.23(7.5 \mathrm{E}-4)$ & $0.996(0.014)$ & $1.11 \mathrm{E}-5(0.0011)$ & $0.089(1.57)$ & $1.06 \mathrm{E}-3(1.5 \mathrm{E}-3)$ & $5.24(101.8)$ & $2.57(2043.2)$ \\
\hline \multirow{4}{*}{1} & 0 & $2.86(0.025)$ & $0.22(7.5 \mathrm{E}-4)$ & $0.996(5.14 \mathrm{E}-06)$ & 9.94E-6(0.16E-3) & $0.090(1.58)$ & $9.9 \mathrm{E}-4(1.6 \mathrm{E}-3)$ & $5.24(102.5)$ & $2.58(1934.8)$ \\
\hline & 1 & $2.86(0.025)$ & $0.24(8.0 \mathrm{E}-4)$ & $0.996(0.08 \mathrm{E}-2)$ & $1.15 \mathrm{E}-5(0.16 \mathrm{E}-3)$ & $0.089(1.58)$ & $1.08 \mathrm{E}-3(0.5 \mathrm{E}-3)$ & $5.20(102.1)$ & $2.33(1988.7)$ \\
\hline & 3 & $2.86(0.025)$ & $0.22(8.2 \mathrm{E}-4)$ & $0.996(0.03 \mathrm{E}-2)$ & $1.04 \mathrm{E}-5(0.20 \mathrm{E}-3)$ & $0.089(1.57)$ & $1.01 \mathrm{E}-3(0.37 \mathrm{E}-3)$ & $5.23(102.2)$ & $2.63(1997.5)$ \\
\hline & 10 & $2.86(0.025)$ & $0.23(7.7 \mathrm{E}-4)$ & $0.996(0.23 \mathrm{E}-2)$ & 1.11E-5(9.9E-5) & $0.089(1.58)$ & $1.06 \mathrm{E}-3(1.2 \mathrm{E}-3)$ & $5.24(102.7)$ & $2.57(2128.5)$ \\
\hline
\end{tabular}

MIT Arrhythmia database [15] using different combinations of $K$-factors $(K=0,1,3,10)$ and normalized distances $(d / \lambda=0.01,0.05,0.1,1)$. The database is obtained from 47 subjects and contains 48 half-hour excerpts that were digitized at 360 samples per second per channel with 11-bit resolution over a $10 \mathrm{mV}$ range. The secure CS measurement matrix is generated once for each record. Thus, MI and other metrics (RE, RMSE, SC) are also evaluated per ECG recording. The size of the matrix is $128 \times 256$ which defines a compression ratio of $50 \%$. It also determines the LFSR seed length $m=15$. The polynomial of our choice is $x^{15}+x^{14}+1$. Daubechies db10 lv4 transform is used as a sparsifying basis $\Psi$ [21]. The choice of the number of steps $L$ in Sensing Matrix Generation block is a trade-off between system security strength and latency. In the simulation, $L$ is chosen to be 10 . This gives 150 random bits extracted from the channel which is sufficient to prevent a brute force attack. Reconstruction problem (3) is solved by the CVX package [22].

3) Experimental Setup: The experiment is of similar design as described in [18] and includes extracting secret bits from the wireless channel. This corresponds to a single Secret Bits Distillation block in Fig. 2. Extracted bits are taken offline for generation of CS sensing matrices which are then applied to the same MIT database as in simulation. For consistency, all applicable parameters from simulation retain their values for the experiment. The experimental study complements our simulation and provides real-world validation of our approach.

In the experiment, we use a TeloSB platform that includes a Chipcon CC2420 RF Transceiver and a TI MSP430 microcontroller. The transceiver is IEEE 802.15.4 compliant and operates at a data rate of $250 \mathrm{kbps}$ in the 2.4 to $2.835 \mathrm{GHz}$ ISM band. A packet transfer protocol is designed to have 4 octet carrying Address, RSSI and Sequence Number. The TeloSB supports the open source operating system TinyOS version 2.1.1. Along with IEEE 802.15.4, TinyOS implements the WPLS protocol extending the total message length to 38 octets. The resulted transmission time per packet is $1.2 \mathrm{~ms}$.

The test is performed while a test subject walks randomly around a typical residential environment which includes a 20'x13.5' living room, 15'x10' and 18'x12' bedrooms, a kitchen and a bathroom. The test subject is a 29 year old man weighing $92.5 \mathrm{Kg}$ and standing 1.86 meters tall. The experiment considers key extraction in close proximity of a sensor node, an AP and Eve. The sensor node is placed on the sternum of the test subject. The AP and Eve are placed 8 inches to the right and to the left of the sensor node respectively. The test lasts for 10,000 message exchanges.

As opposed to simulation, consecutive RSSI estimates in the experiment are correlated. To eliminate this problem, we employ a simple distillation algorithm presented in [18]. For clarity and convenience we now briefly describe the algorithm. For every received packet, RSSI value is extracted and the difference between the current and the previous values is calculated. The absolute sum of $Q$ differences $|S|$ is then compared to a predefined threshold $\beta$. When the absolute sum exceeds the threshold, i.e. $|S|>\beta$, a secret bit is generated by taking the sign of $S$. After multiple sets of $Q$ differences, legitimate communicating parties exchange the indices of those sets who passed the threshold. This facilitates the agreement process and allows excluding the bits generated by only one of the parties. Note that it is very unlikely for Eve to generate the same bit sequence due to different RSSI estimates. Following the encryption block-diagram in Fig. 2, the generated binary sequence is used to generate a CS sensing matrix that is applied to ECG database in the same manner as was done in simulation. The values of $Q$ and $\beta$ are set to achieve a reliable key extraction with appropriate statistical properties. From [18] it follows that values of $Q=8$ and $\beta=10$ are suitable for our purposes since the corresponding binary sequence is of sufficient length and passes all applicable NIST randomness tests [23].

\section{B. Complexity and Computing Cost}

We analyze the complexity of the proposed encryption framework relative to the reference framework. The proposed method is essentially the one given in Fig. 2. In the heart of the reference method is the AES block that encrypts data after 
TABLE II

RECONSTRUCTION OF AP AND EVE FOR $Q=8$ AND $\beta=10$

\begin{tabular}{c|l|l} 
& mean & variance \\
\hline MI & $2.86(0.02)$ & $0.22(7.27 \mathrm{E}-04)$ \\
SC & $0.996(0.01)$ & $1.12 \mathrm{E}-05(3.04 \mathrm{E}-5)$ \\
RE & $0.089(1.58)$ & $0.0011(6.0 \mathrm{E}-5)$ \\
RMSE & $5.2(102.5)$ & $2.5(2011.2)$
\end{tabular}

compression with CS takes place. For consistency, proposed and reference frameworks use the same WPLS algorithm for extracting the encryption/decryption key. In practice, decryption is done on a more powerful device compared to a sensornode and therefore only encryption process is analyzed.

In the analysis we use the following notations. $S_{C S}$ number of samples being processed in a single step of CS, $S_{A E S}$ - number of bits being processed in a single step of AES, $T_{C S}$ and $T_{A E S}$ - processing time of a single step for CS and AES respectively, $T_{W P L S}$ - time required to generate a random bit sequence of approximately 128 bits, $T_{L F S R}$ - time required to generate a m-sequence using LFSR, $T_{S M G}$ - time required to generate a sensing matrix having $L \mathrm{~m}$-sequences, $\alpha$ - CS compression ratio, $q$ - number of bits per sample.

Thus, time $T_{\text {prop }}$ to encrypt $N_{B}$ blocks of $S_{C S}$ samples each using the proposed model consists of extraction of a random sequence of bits $\left(T_{W P L S}\right)$, generation of $L \mathrm{~m}$-sequences $\left(L \times T_{L F S R}\right)$, formation of a sensing matrix $\left(T_{S M G}\right)$ and CS execution for each block $\left(N_{B} \times T_{C S}\right)$ :

$$
T_{\text {prop }}=T_{W P L S}+L \times T_{L F S R}+T_{S M G}+N_{B} \times T_{C S}
$$

Time $T_{\text {ref }}$ to encrypt same $N_{B}$ blocks using the reference model consists of key generation $\left(T_{W P L S}\right)$, execution of CS for all $N_{B}$ blocks $\left(N_{B} \times T_{C S}\right)$ and execution of AES $\left\lceil S_{C S} \times q \times \alpha / S_{A E S}\right\rceil$ times per each block:

$$
T_{r e f}=T_{W P L S}+N_{B} \times\left(T_{C S}+\left\lceil\frac{S_{C S} \times q \times \alpha}{S_{A E S}}\right\rceil T_{A E S}\right)
$$

Relative time complexity of the proposed scheme can be expressed as a ratio of (4) and (5). As the number of blocks to be encrypted increases, relative complexity converges to:

$$
\rho=\lim _{N_{B} \rightarrow \infty} \frac{T_{\text {prop }}}{T_{r e f}}=\frac{T_{C S}}{T_{C S}+\left\lceil\frac{S_{C S} \times \alpha \times q}{S_{A E S}}\right\rceil T_{A E S}}
$$

\section{Security Strength}

MI quantifies how much information $\hat{x}_{S E}$ carries about $x_{S}$, i.e. whether the framework is secure in presence of an eavesdropper in close vicinity. More specifically, we use a JamesStein (JS) shrinkage estimator of MI presented in [16]. The estimator exhibits high computational efficiency and is reliable in case of undersampled data. JS cell frequencies are estimated as $\hat{\Theta}_{k}=\lambda t_{k}+(1-\lambda) \hat{\Theta}_{k}^{M L}$, where $\lambda \in[0,1]$ is the shrinkage intensity, $t_{k}$ is the shrinkage target distribution which is set to uniform, and $\hat{\Theta}_{k}^{M L}$ is the corresponding maximum likelihood frequencies. MI is found for both simulation and experimental setups the same way it was done for quality of reconstruction. For estimation of MI, the reconstructed and original signals are quantized to 8 bits and scaled to have identical finite set of possible values. In addition to evaluating MI between Eve and the sensor node, we also evaluate MI between the AP and the sensor node to make sure reconstruction is successful.

In the rest of this section we present security strength of the proposed framework when basic cryptographic attacks are applied. Since the order in which the seeds are extracted is irrelevant for sensing matrix generation, the complexity of the basic brute force attack is diminished by the number of permutations of $L$ and is given by $2^{m \times L} / L$ !. For $L=10$ and $m=15$ this results in enormous computational efforts $\left(2^{m \times L} / L !>2^{128}\right)$. However, Eve can substantially reduce the complexity by exploiting proximity to a legitimate receiver. For example, if Eve is close to the AP then their wireless channels are highly correlated meaning that the random sequence she extracts is very similar to the one used in legitimate communication. In other words, distance from the AP helps the attacker deduce how many bits need to be flipped in order to get the correct key. If Eve knows that the corresponding distance results in approximately $h$ different bits then the complexity of this attack is described as the number of combinations $\left(\begin{array}{c}m \times L \\ h\end{array}\right)$.

Initially, we find a minimum Hamming distance that makes combinatorial search impractical just as in the case of AES, i.e. of the same complexity as $2^{128}$. In the next step, we perform simulation experiments to investigate the behavior of the Hamming distance between Eve's and AP's distilled binary sequences for different combinations of normalized distances $(d / \lambda=0.01,0.02, \ldots, 2)$ and $K$-factors $(K=0,1,3,10)$. At each step, the average Hamming distance over 1000 realizations is calculated. All other applicable parameters reported in section IV-A2 remain the same. Results help to reveal how far Eve needs to be in order to achieve the minimum required Hamming distance that was found previously.

Another security aspect that needs careful consideration is how sensitive a measurement matrix is to small variations in an underlying binary sequence and how it affects the quality of reconstruction. If two similar sequences result in similar measurement matrices then it is not necessary for Eve to get the exact key. This allows Eve to simplify the exhaustive search. To measure the similarity between sensing matrices we use a relative perturbation metric $\epsilon=\left\|\Phi-\Phi^{\prime}\right\|_{2} /\|\Phi\|_{2}$, where $\Phi$ and $\Phi^{\prime}$ are measurement matrices generated from original seed and similar to it respectively. Thorough theoretical analysis of effect of relative perturbation on the reconstruction process was provided in [24]. In our simulation, for every combination of a Hamming distance from a set $[1,3,5,7,10,13,15]$ and a number of steps in sensing matrix generation $L$ in the range [1 - 50] the average $\epsilon$ over 100 random is computed. All applicable simulation parameters are identical to IV-A2.

\section{RESULTS AND DISCUSSION}

\section{A. Quality of Reconstruction}

Simulation results are reported in Table I. Each entry in the table is an average value over 48 records. Corresponding results for an adversary are given in parentheses next to values for legitimate participants (AP and sensor node). Similarity metrics of SC, RE, RMSE confirm that for all tested scenarios 


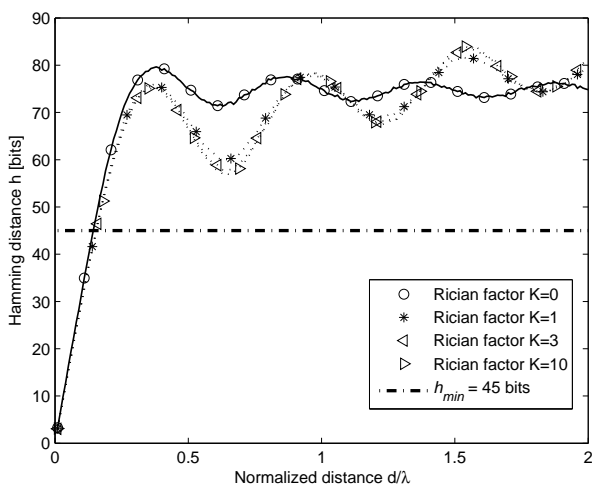

Fig. 4. Hamming distance for different combinations of $d / \lambda$ and $K$

the signal reconstructed by Eve on average cannot be used for practical purposes. Reconstruction examples for Eve and the AP is presented in Fig. 6(b) and Fig. 6(d) respectively. It is obvious that the signal recovered by Eve does not resemble the original pulse. At the same time, the legitimate party meets or exceeds commonly used benchmark values. Thus, SC has an average value of 0.996 whereas the acceptable values is known to be greater than 0.9 [19]. Besides, SC exhibits extremely low variation which attests to the consistency of the results. The same applies to RE and RMSE. Their average values and variance also conform with Fig. 6 and exhibit almost perfect reconstruction at the AP and completely unacceptable for Eve. The overall trend of the table is as follows: as distance increases, Eve's ability to reconstruct the data is diminishing. Her mean values become farther and farther away from the correct values while variance is getting smaller.

The experimental setup places Eve 8 inches away from the sensor node. Along with communication frequency of $2.4 \mathrm{GHz}$ this gives an approximate values of $d / \lambda=1$.6. Experimental results are reported in Table II. As in Table I, corresponding values for Eve are given in parentheses. It is seen that the experimental results are almost identical to simulation and provide near perfect average values with low variance of all metrics for AP. Eve experiences extremely poor average and low variance without adequate reconstruction.

Similar experiment was done in the residential environment with the subject sitting down for $L$ in the range of $[1-20]$. Results showed that the proposed matrices behave similarly to pseudorandom gaussian matrices for all tested $L$ and give the same trend as reported in Table II.

\section{B. Complexity and Computing Cost}

Relative time complexity showed in (6) implies that $\rho<$ 1 meaning that the complexity of the proposed encryption framework is always lower. For example, using typical parameter values $\left(S_{C S}=256\right.$ samples, $\alpha=0.5, q=11$ bits, $S_{A E S}=128$ bits) results in relative complexity $\rho=$ $T_{C S} /\left(T_{C S}+11 \times T_{A E S}\right)$ which gives a time advantage of not needing to process 11 AES blocks. On a 64-bit core i7 machine with $8 \mathrm{~Gb}$ of RAM running Windows 7, Matlab implementation of AES from [25] results in $T_{A E S} \approx 2 \mathrm{~ms}$,

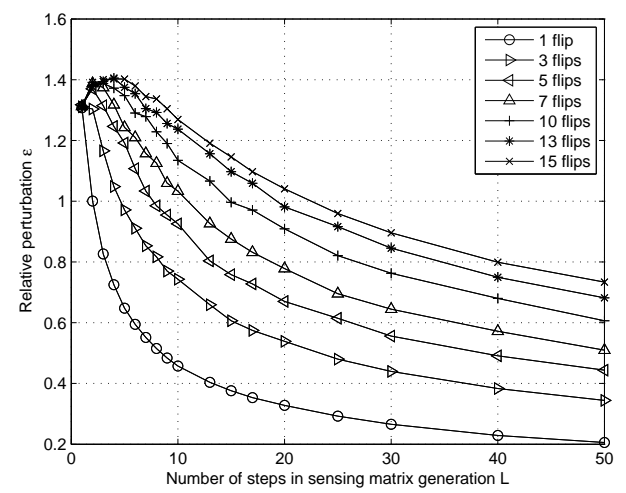

Fig. 5. Relative perturbation for different number of flipped bits

whereas $T_{C S} \approx 0.02 \mathrm{~ms}$. This yields $\rho \approx 0.9 \times 10^{-3}$. Although Matlab would not be implemented on a sensor-node, this number gives an idea of the difference in computing costs.

\section{Security Strength}

Estimated MI is given in tables I and II for simulation and experimental results respectively. In both cases, we observe the same trend as for other similarity metrics. MI between the original signal and the signal reconstructed by the AP is always high and has a value of 2.86 bits on average. At the same time Eve's reconstruction is poor. When $d / \lambda$ is low $\approx$ 0.1 the wireless channel does not provide sufficient spatial selectivity and there are cases when Eve is able to extract the measurement matrix equal to the one extracted by the AP or the sensor node. This fact is reflected in higher average values and higher variance of MI. The low variance of Eve's as well as AP's MI implies that Eve's reconstruction is consistently erroneous while the AP's is stable and accurate.

Analyzing the number of combinations $\left(\begin{array}{c}m \times L \\ h\end{array}\right)$ we conclude that the minimum Hamming distance $h_{\text {min }}$ required to prevent combinatorial search is 45 bits assuming $L=10$ and $m=15$. Variation of the Hamming distance with normalized distance and multiple $K$-factors is presented in Fig. 4. Ripple behavior is due to correlation properties of the Rician channel and is expected. If Eve is located farther than $d / \lambda=0.16$ away from the AP then the system is secure since the Hamming distance is always above $h_{m i n}$. Rician factor has only marginal effect on security strength in the considered scenario.

Sensitivity of a sensing matrix in terms of relative perturbation is presented in Fig. 5. As the number of steps used in sensing matrix generation increases the relative perturbation decreases. This result is expected since for high $L$ smaller subset of its random bits gets affected. For fixed $L>1$ relative perturbation becomes stronger according to the number of flipped bits. Fig. 6(a-c) shows that even in the worst case scenario for Haming distance $h=1$, Eve cannot obtain proper reconstruction achievable for $\epsilon<0.05$ [24].

\section{CONCLUSION}

In this paper, we proposed a framework for securing wireless links in biomedical applications involving WBANs. The 

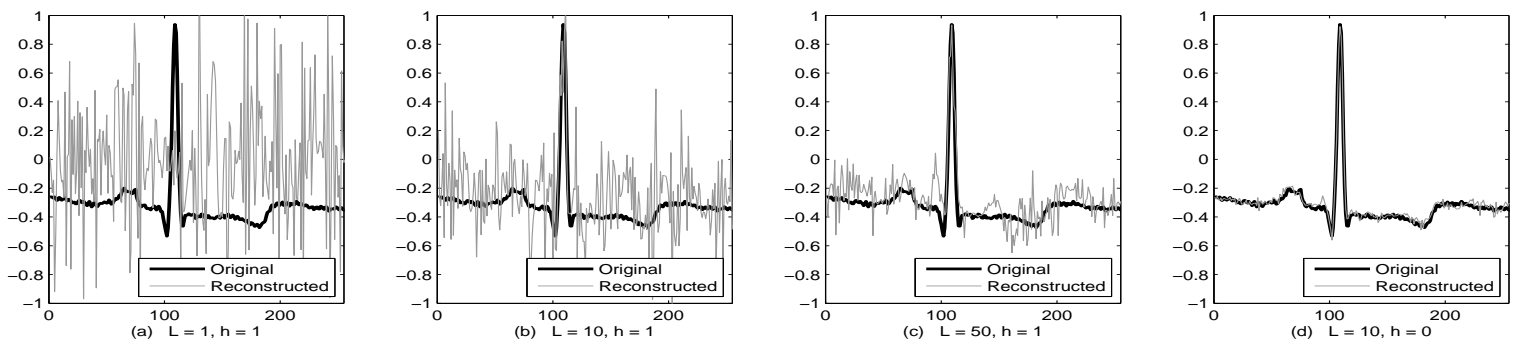

Fig. 6. Example of reconstruction of a single pulse from record $100 \mathrm{~m}$ for different number of steps $L$ in matrix generation process $(K=3, d / \lambda=1)$

framework relies on using a CS measurement matrix as a symmetric key for encryption and decryption. The matrix is securely generated for both sensor-node and an access point using low complexity WPLS algorithm. The encryption becomes an integral part of the sampling process eliminating the need for a separate encryption algorithm and substantially minimizing computational and memory requirements critical for WBANs. The framework was evaluated in the presence of an eavesdropper performing a proximity attack and applied to 48 ECG records of MIT Arrhythmia database. Both simulations and experiment methodologies that reflect a wireless ECG monitoring scenario were employed for testing. Simulation assumed Rician fading channel and provided control on a normalized distance and Rician $K$-factor. Experiment extracted bits from the wireless channel and included a sensor node, an access point and Eve on the test subject's body where Eve was located 8 inches away from the sensor node.

Various similarity metrics SC, RE, RMSE verified that while an AP achieves accurate reconstruction with near optimal average values and low variance, Eve's reconstruction is extremely poor and useless for any practical purposes. Using the mutual information and relative perturbation metrics, we showed that the proposed framework generates strong keys and is resistant against an eavesdropper located at a reasonable distance. Further security analysis concluded that distances larger than $d / \lambda=0.16$ from legitimate participants is safe. This allows for practical consideration and gives a distance of 2 centimeters assuming $2.4 \mathrm{GHz}$ communication frequency. Through relative time complexity analysis it was shown that the proposed encryption framework is much faster than its state-of-the-art competitor based on AES.

\section{REFERENCES}

[1] Centers for Medicare \& Medicaid Services, "The Health Insurance Portability and Accountability Act of 1996 (HIPAA)," Online at http://www.cms.hhs.gov/hipaa/, 1996.

[2] J. Daemen and V. Rijmen, The Design of Rijndael: AES - The Advanced Encryption Standard, ser. Information Security and Cryptography. Springer, 2002.

[3] W. Diffie and M. Hellman, "New directions in cryptography," IEEE Trans. Inf. Theor, vol. 22, no. 6, pp. 644-654, Sep. 2006.

[4] E. J. Candes, J. Romberg, and T. Tao, "Robust uncertainty principles: Exact signal reconstruction from highly incomplete frequency information," IEEE Trans. Inf. Theor., vol. 52, no. 2, pp. 489-509, Feb. 2006.

[5] E. J. Candes and T. Tao, "Near-optimal signal recovery from random projections: Universal encoding strategies?" IEEE Trans. Inf. Theor., vol. 52, no. 12, pp. 5406-5425, Dec. 2006.

[6] D. L. Donoho, "Compressed sensing," IEEE Trans. Inform. Theory, vol. 52, pp. 1289-1306, 2006.
[7] M. R. Mayiami, B. Seyfe, and H. G. Bafghi, "Perfect secrecy using compressed sensing," CoRR, vol. abs/1011.3985, 2010.

[8] Y. Rachlin and D. Baron, "The secrecy of compressed sensing measurements," 2008 46th Annual Allerton Conference on Communication, Control, and Computing, pp. 813-817, Sep. 2008.

[9] G. Reeves, N. Goela, N. Milosavljevic, and M. Gastpar, "A compressed sensing wire-tap channel," CoRR, vol. abs/1105.2621, 2011.

[10] J. W. Wallace and R. K. Sharma, "Automatic secret keys from reciprocal mimo wireless channels: measurement and analysis." IEEE Transactions on Information Forensics and Security, vol. 5, no. 3, pp. 381-392, Sept 2010.

[11] C. Ye, S. Mathur, A. Reznik, Y. Shah, W. Trappe, and N. B. Mandayam, "Information-theoretically secret key generation for fading wireless channels," IEEE Transactions on Information Forensics and Security, vol. 5, pp. 240-254, June 2010.

[12] G. R. Tsouri and D. M. Wagner, "Threshold constraints on symmetric key extraction from rician fading estimates." IEEE Trans. Mob. Comput., vol. 12, no. 12, pp. 2496-2506, 2013.

[13] Y. Liang, H. V. Poor, and S. Shamai (Shitz), "Secure Communication Over Fading Channels," IEEE Transactions on Information Theory, vol. 54, no. 6, pp. 2470-2492, Jun. 2008.

[14] R. Dautov and G. R. Tsouri, "Establishing secure measurement matrix for compressed sensing using wireless physical layer security," in Computing, Networking and Communications (ICNC), 2013 International Conference on. IEEE, 2013, pp. 354-358.

[15] A. L. Goldberger, L. A. N. Amaral, L. Glass, J. M. Hausdorff, P. C. Ivanov, R. G. Mark, J. E. Mietus, G. B. Moody, C.-K. Peng, and H. E. Stanley, "PhysioBank, PhysioToolkit, and PhysioNet: Components of a New Research Resource for Complex Physiologic Signals," Circulation, vol. 101, no. 23, pp. e215-e220, Jun. 2000.

[16] J. Hausser and K. Strimmer, "Entropy inference and the James-Stein estimator, with application to nonlinear gene association networks," The Journal of Machine Learning Research, vol. 10, pp. 1469-1484, 2009.

[17] P. S. Addison, "Wavelet transforms and the ecg: a review," Physiological measurement, vol. 26, no. 5, p. R155, 2005.

[18] G. R. Tsouri and J. Wilczewski, "Reliable symmetric key generation for body area networks using wireless physical layer security in the presence of an on-body eavesdropper," Proceedings of the 4th International Symposium on Applied Sciences in Biomedical and Communication Technologies - ISABEL '11, pp. 1-6, 2011.

[19] B. M. Horáček, J. W. Warren, and J. J. Wang, "On designing and testing transformations for derivation of standard 12-lead/18-lead electrocardiograms and vectorcardiograms from reduced sets of predictor leads," Journal of Electrocardiology, vol. 41, no. 3, pp. 220-229, 2008.

[20] C. Xiao, Y. R. Zheng, and N. C. Beaulieu, "Novel sum-of-sinusoids simulation models for rayleigh and rician fading channels." IEEE Transactions on Wireless Communications, vol. 5, no. 12, pp. 3667 3679, 2006.

[21] I. Daubechies et al., Ten lectures on wavelets. SIAM, 1992, vol. 61.

[22] M. Grant and S. Boyd, "CVX: Matlab software for disciplined convex programming, version 2.0 beta," http://cvxr.com/cvx, Sep. 2013.

[23] A. Rukhin, J. Soto, J. Nechvatal, M. Smid, and E. Barker, "A statistical test suite for random and pseudorandom number generators for cryptographic applications," DTIC Document, Tech. Rep., 2001.

[24] M. A. Herman and T. Strohmer, "General deviants: An analysis of perturbations in compressed sensing," Selected topics in signal processing, IEEE Journal of, vol. 4, no. 2, pp. 342-349, 2010.

[25] J. Buchholz. (2001, Dec.) Matlab implementation of the advanced encryption standard @ONLINE. [Online]. Available: http://buchholz.hsbremen.de/aes/aes.htm 